\title{
Bolus Calculator Reduces Hypoglycemia in the Short Term and Fear of Hypoglycemia in the Long Term in Subjects with Type 1 Diabetes (CBMDI Study)
}

\author{
María del Rosario Vallejo Mora, PhD, MD, ${ }^{1,2}$ Mónica Carreira, PhD, 2,3 \\ María Teresa Anarte, $\mathrm{PhD}_{1}^{2,3}$ Francisca Linares, $\mathrm{MD}_{1}^{2,4}$ \\ Gabriel Olveira, PhD, MD, ${ }^{1,2,4}$ and Stella González Romero, PhD, MD ${ }^{1,2,4}$
}

\begin{abstract}
Background: In a previous study we demonstrated improvement in metabolic control and reduction in hypoglycemia in people with type 1 diabetes on multiple daily injections, after having used a bolus calculator for 4 months. Objective: To demonstrate whether (1) extending its use (2) or introducing it in the control group, previously subjected to treatment intensification, could further improve metabolic control and related psychological issues. Methods: After the previous clinical trial, in which the subjects were randomized either to treatment with the calculator or to control group for 4 months, both groups used the calculator during an additional 4-month period.

Results: In the previous control group, after using the device, HbA1c did not improve $(7.86 \% \pm 0.87 \%$ vs. $8.01 \% \pm 0.93 \%, P 0.215)$, although a significant decrease in postprandial hypoglycemia was observed $(2.3 \pm 2$ vs. $1.1 \pm 1.2 / 2$ weeks, $P$ 0.002). In the group in which the treatment was extended from 4 to 8 months, HbA1c did not improve either $(7.61 \pm 0.58$ vs. $7.73 \pm 0.65, P 0.209)$; however this group had a greater perceived treatment satisfaction $(12.03 \pm 4.26$ vs. $13.71 \pm 3.75, P 0.007)$ and a significant decrease in fear of hypoglycemia $(28.24 \pm 8.18$ basal vs. $25.66 \pm 8.02$ at 8 months, $P$ 0.026).

Conclusions: The extension in the use of the calculator or its introduction in a previously intensified control group did not improve metabolic control, although it did confirm a decrease in hypoglycemic episodes in the short term, while the extension of its use to 8 months was associated with a reduction in fear of hypoglycemia and greater treatment satisfaction.
\end{abstract}

Keywords: Type 1 diabetes, Bolus calculator, Multiple daily injections of insulin, Hypoglycemia, Psychology.

Highlights

- In people with type 1 diabetes, on a previously intensified treatment, we confirm that the use of a bolus calculator can reduce the hypoglycemic episodes for the short term.

- For the long term (8 months) a bolus calculator does not improve further the overall metabolic control, but some psychological aspects, such as the fear of hypoglycemia and satisfaction with the treatment, can still get better.

\section{Introduction}

TN SUBJECTs WITH type 1 diabetes (DM1) on treatment intensification, either with multiple daily injections of insulin (MDI) or a continuous subcutaneous insulin infusion (CSII), it is necessary to properly calculate the insulin bolus, both for prandial and corrective insulin. That calculation includes variables such as carbohydrate count $(\mathrm{CH})$, management of insulin/ $\mathrm{CH}$ ratio $(\mathrm{CarbF})$, or insulin sensitivity factor (ISF), all of which require personalization and can be

\footnotetext{
${ }^{1}$ Endocrinology and Nutrition Department, Hospital Regional Universitario de Málaga, Málaga, Spain.

${ }^{2}$ Instituto de Investigación Biomédica (IBIMA), Hospital Regional Universitario de Málaga, Málaga, Spain.

${ }^{3}$ Personality, Evaluation and Psychological Treatment, School of Psychology, Málaga Spain.

${ }^{4}$ CIBER of Diabetes and Metabolic Diseases (CIBERDEM), Barcelona, Spain.
}

(c) María del Rosario Vallejo Mora, et al., 2017; Published by Mary Ann Liebert, Inc. This Open Access article is distributed under the terms of the Creative Commons Attribution Noncommercial License (http://creativecommons.org/licenses/by-nc/4.0/) which permits any noncommercial use, distribution, and reproduction in any medium, provided the original author(s) and the source are credited. 
complicated. A published study demonstrated that people with DM1 on treatment with MDI did not correctly calculate the majority of bolus. ${ }^{1}$ The use of bolus calculators (BCs) allows the patient to obtain a more precise and personalized recommended insulin dose by means of a preprogrammed system. In people with diabetes on treatment with CSII, this is already part of the standard clinical practice. The introduction of BCs for people on treatment with MDI, combined with blood glucose monitors or apps for smartphones, is relatively recent. With the use of $\mathrm{BCs}$, the subject depends on a device to help him make decisions concerning the treatment, which involves a process of learning, adaptation, and the building of confidence, all of which may involve added stress.

The BC contribution to the improvement of metabolic control and its impact on psychological issues such as fear of hypoglycemia and level of treatment satisfaction is documented in recent publications. ${ }^{2-6}$ We will only quote studies performed in people on MDI (not CSII) treatment. Two randomized clinical trials (CTs) are compulsory references, both performed with the Accu-Chek ${ }^{\circledR}$ Aviva Expert device: (1) The BolusCal study, ${ }^{4}$ in which in both intervention groups (measuring $\mathrm{CH}$, with or without the use of a $\mathrm{BC}$ ) an improvement in HbA1c was observed $(-0.8 \%$ and $-0.7 \%$ reduction, respectively), although without significant differences between them. There were no differences between groups regarding hypoglycemic episodes. (2) The ABACUS (Automated Bolus Advisor Control and Usability Study) revealed that more patients who used a $\mathrm{BC}$ managed to reduce their $\mathrm{HbA} 1 \mathrm{c}>0.5 \%$ compared with the control group $(56 \%$ vs. $34.4 \% ; P<0.001)$; however, an increase in hypoglycemia $(<70 \mathrm{mg} / \mathrm{dL})$ was found. ${ }^{5,7-9}$

A recent publication, the Expert study, shows how the use of a BC also allowed a significant reduction in HbA1c (although similar to the one obtained in the active control group) associated with a decrease in postprandial hypoglycemia $(<60 \mathrm{mg} / \mathrm{dL}){ }^{6}$

With reference to the psychological evaluation, at the end of the BolusCal study the people who used the BC obtained better scores on treatment satisfaction, with no significant differences observed with regard to fear of hypoglycemia. ${ }^{4}$ In the ABACUS study a significant improvement was also noted in treatment satisfaction compared with the control group; however the evaluation of the remaining psychological variables, such as fear of hypoglycemia, has not been published yet. ${ }^{5,7-9}$ In the Expert study a significant improvement in quality of life and satisfaction with the use of BC was observed. ${ }^{6}$

Recently we have published the results of a CT in which subjects were randomized to use a $\mathrm{BC}$ or to a control group for 4 months. ${ }^{10}$ Both groups were active in the handling of concepts related with prandial bolus calculation $(\mathrm{CH}$ measuring, CarbF, ISF, etc.), although only one of them used the device. We observed a significant decrease in HbAlc compared with baseline in both groups. A significant decrease in the total number of hypoglycemic episodes was found only in the group that used the BC. There were no significant differences regarding fear of hypoglycemia or treatment satisfaction. ${ }^{10}$

The aim of this article is to evaluate whether the extension of the treatment with BC (from 4 to 8 months) or the introduction of it in the previously intensified control group could mean an additional improvement in glycemic control or hypoglycemic episodes or changes in the related psychological issues.

\section{Methods}

The participants in this study came from a clinical trial (CBMDI study, Calculating Boluses on Multiple Daily Injections) performed by the Endocrinology and Nutrition Department, Hospital Regional Universitario de Málaga, which evaluated the metabolic and psychological effects of the incorporation of a BC in DM1 people on MDI treatment. The study, including this extension, was approved by the Investigation Ethics Committee of the Hospital Regional Universitario of Málaga, and conforms to the provisions of the Declaration of Helsinki. The patients had been randomized to use a $\mathrm{BC}$ ( $\mathrm{Cb}$ group) or to be in the active control group (Co group) during 4 months. People with DM1 > 18 years old, with $\mathrm{HbAlc}>7 \%$, on treatment with basal-bolus therapy, and not having suffered from more than two severe hypoglycemic episodes in the precedent year were included. We used the device Accu-Chek Aviva Expert BC (Roche Diagnostics, Indianapolis, IN). The results presented in this study were obtained from the extension of the previous study for a further 4 months, in which all participants were joined in a single group, all of them using the $\mathrm{BC}$. The control group (Co group), which was starting using the $\mathrm{BC}(\mathrm{Co} \rightarrow \mathrm{Cb}$ group), was trained in its use in the last visit of the previous phase of the study, and had, as was previously done with the $\mathrm{Cb}$ group, an additional visit 2 weeks afterward to ensure the correct use of the device. Subsequently both groups had two scheduled visits, after 2 and 4 months. The same protocol was followed in both visits as in the previous study (educational support, downloading the self-monitoring of blood glucose [SMBG] from the 2 weeks before the visit, adjustment of the basal insulin dose, and modification of the programming of CarbF and ISF). Furthermore, the tools of psychological evaluation were applied, and an analytical extraction was made at the start and end of the second phase (fourth and eighth month).

The variables initially collected were sex, age, years of diabetes evolution, and education level. With regard to metabolic control, we evaluated $\mathrm{HbA} 1 \mathrm{c}$ and its modifications, and from the 2 weeks before each visit (through downloaded SMBG registers) we checked mean glycemia (total, pre- and postprandial) and number of major hyperglycemia $(>250 \mathrm{mg} / \mathrm{dL})$ and hypoglycemia ( $<70$ and $<50 \mathrm{mg} / \mathrm{dL}$, pre- and postprandial and nocturnal) episodes; we also recorded the number of severe and moderate hypoglycemic events [defined, respectively, as (1) severe: loss of consciousness, having used glucagon, or requiring professional healthcare attention; (2) moderate: with the need of help from a third person, but resolved with oral glucose] for all the study period. Estimations of glycemic variability were also calculated (SD, CV, MAGE, LBGI, and HBGI).

Unless the participant was using a personalized CarbF and/ or ISF before the beginning of the study, these factors were established, respectively, in $1 \mathrm{IU} / \mathrm{KE}$ and $50 \mathrm{mg} /(\mathrm{dL} \cdot \mathrm{IU})$.

The changes in CarbF (IU/KE) and ISF (mg/dL of glycemia reduced by 1 extra IU) throughout the study were likewise recorded.

A questionnaire was included, as in the first phase of the study, to show the frequency of use and acceptance of the BC (basal, 4 and 8 months). The questionnaire was adapted from the "Device Utilization by the Patient" from the ProAct study ${ }^{11}$ with the authorization of Roche Diabetes Care. It consists of nine questions with a score of answers ranging from 1 to 5 ( 5 being the highest score). 


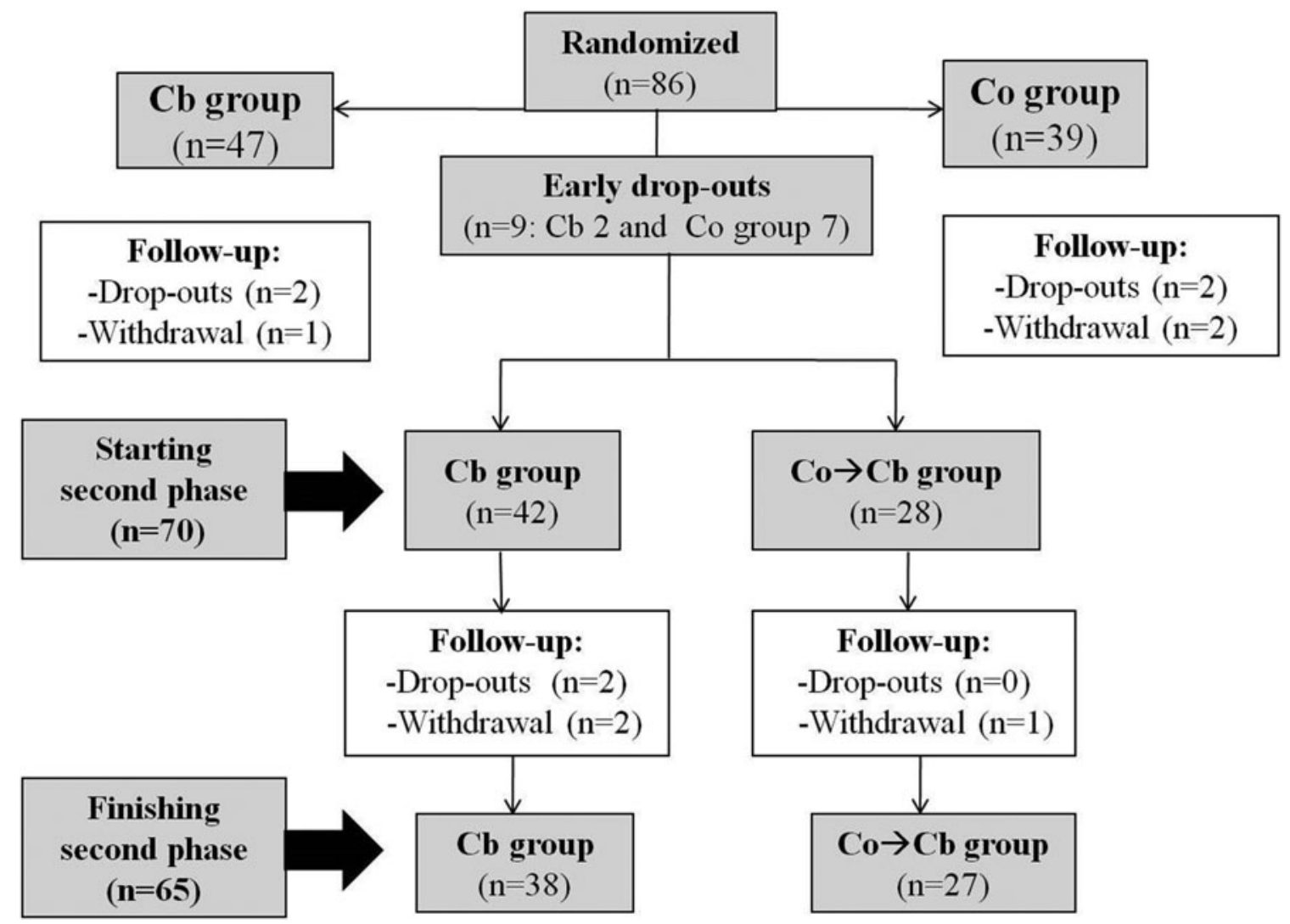

FIG. 1. Flow chart of subjects during the study.

Treatment satisfaction was evaluated by means of the Spanish version ${ }^{12}$ of the Diabetes Treatment Satisfaction Questionnaire (DTSQ), ${ }^{13}$ with two versions to be completed, at the start (DTSQs) and after the treatment/follow-up (DTSQc). A higher score represents a higher level of satisfaction. The participants also fulfilled the Fear of Hypoglycemia FH-15 questionnaire, ${ }^{14}$ which consists of 15 items with an answer scale ranging between 1 and 5 . A sum of scores $\geq 28$ is considered indicative of fear of hypoglycemia.

\section{Statistical study}

The sample size was calculated for the previous CT, having being estimated at 82 subjects. ${ }^{10}$ The sample of the present study stems from the acceptance of the participants to extend the previous study.

We present the results of an intention to treat analysis. Continuous variables were summarized using mean and SD values; categorical variables were summarized using counts and percentages of patients in each category. To test hypothesis, the Student's $t$ test for continuous variables with normal distribution, nonparametric test (Mann-Whitney or Wilcoxon) in the case of continuous variables that did not conform to normal, and the $\chi^{2}$ for the association between proportions were performed. To check changes over time, the McNemar test was used for proportions, and the one way ANOVA with repeated measures was used for continuous variables. Linear regression models were performed to explain the relationship of dependence among some variables. The alpha error to reject null hypothesis was 0.05 for two tails.

\section{Results}

In Figure 1 the flow of participants in the study is shown, including the first 4 month randomized CT phase, the results of which have already been published. We are presenting mainly the results of the second phase of the study, this is, from the fourth to the eighth month. Forty-two subjects started this phase coming from the group that had already been using the $\mathrm{BC}$ (Cb group); 28 subjects came from the control group (Co group) and started using the $\mathrm{BC}(\mathrm{Co} \rightarrow \mathrm{Cb}$ group). Sixty-five participants completed the study (38 and 27, respectively).

There were no differences between groups in the general characteristics of the subjects (sex, age, and years of DM evolution) at the start of the second phase of the study (25 females/17 males in Cb group, 13 females/15 males in $\mathrm{Co} \rightarrow \mathrm{Cb}$ group, $P$ 0.281; aged $30.69 \pm 12.19$ years in $\mathrm{Cb}$ group vs. $34.21 \pm 12.09$ years in $\mathrm{Co} \rightarrow \mathrm{Cb}$ group, $P$ 0.239; duration of DM 14.76 \pm 9.79 years in $\mathrm{Cb}$ group vs. $16.18 \pm 8.46$ in $\mathrm{Co} \rightarrow \mathrm{Cb}$ group, $P$ 0.534).

Metabolic control was not different between groups at the beginning of the study; it improved for both groups at the fourth month (published data) and did not improve further during the study extension up to 8 months, either in patients who extended the use of the $\mathrm{BC}$ from 4 to 8 months or in those patients (with previously intensified treatment) to whom the use of the BC was added. HbA1c even looked to increase slightly from 4 to 8 months, although not significantly, and was not different between groups (Table 1). But if we considered the whole sample $(\mathrm{Cb}+\mathrm{Co} \rightarrow \mathrm{Cb})$ and divided it into two groups, this is, participants that just before using $\mathrm{BC}$ had 
Table 1. Metabolic Control: 4-8 Months (Second Phase of the Study)

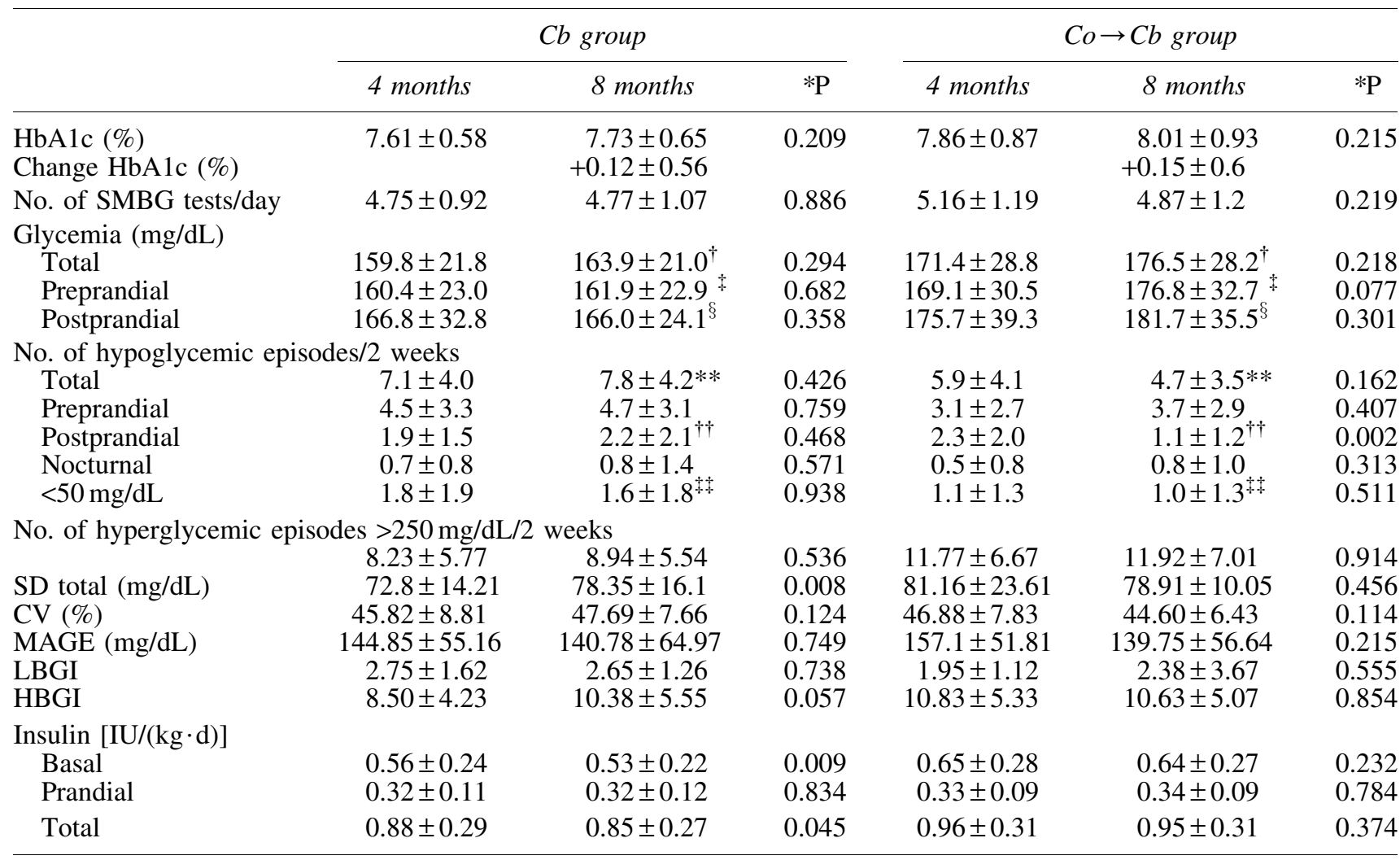

Mean \pm SD.

${ }^{*} P$ intragroups. Between groups at 8 months: ${ }^{\dagger} P 0.045 ;{ }^{\ddagger} P 0.035 ;{ }^{\S} P 0.032 ; * * P 0.003 ;{ }^{\dagger \dagger} P 0.006$; and ${ }^{\dagger} P 0.026$.

$\mathrm{Cb}$, $\mathrm{BC}$ group; Co, control group; $\mathrm{CV}$, coefficient of variation; HBGI, high blood glucose index; LBGI, low blood glucose index; MAGE, mean amplitude of glycemic excursions; SD, standard deviation.

a HbA1c $\geq$ or $<8.5 \%$, they showed a different behavior regarding metabolic control. People having a HbA1c $\geq 8.5 \%$ just before using BC reduced the HbA1c significantly after 4 months of having used it $(9.18 \% \pm 0.67 \%$ vs. $8.29 \% \pm 0.97 \%$, $P<0.001)$. The reduction in the HbAlc for participants with a basal figure $<8.5 \%$ was not significant $(7.63 \pm 0.41$ vs. $7.58 \pm 0.59, P 0.58)$. The same behavior was found regarding HbAlc $\geq$ or $<8 \%$.

The results obtained in a linear regression model, in which the dependent variable was HbAlc at 4 and 8 months, can be seen in the Tables 2 and 3. HbA1c at 4 months (the end of the previous $\mathrm{CT}$ ) could be explained in terms of variables such as age, education level, basal HbAlc, and also the treatment group belonged to (favoring the use of $\mathrm{BC}$ ). In this extension phase, in the multivariate study, only previous HbAlc (4 months) and the number of daily SMBG could be associ-

Table 2. Linear Regression Model HbA1C at 4 Months

\begin{tabular}{lcccccc}
\hline Model & $\mathrm{B}$ & Std err & Beta & $\mathrm{t}$ & $\mathrm{P}$ & Adj $R^{2}$ \\
\hline Basal HbA1c & 0.452 & 0.084 & 0.480 & 5.393 & 0.000 & 0.505 \\
$\begin{array}{l}\text { Education } \\
\quad-0.238\end{array}$ & 0.074 & -0.283 & -3.199 & 0.002 & \\
$\quad$ level & & & & & & \\
$\begin{array}{l}\text { Age (years) } \\
\text { Treatment }\end{array}$ & -0.016 & 0.006 & -0.251 & -2.827 & 0.006 & \\
$\quad$ group & & & & & & \\
$\quad$
\end{tabular}

Sex, SMBG tests at 4 months, No. of hypoglycemic episodes at 4 months, and BMI did not enter in the model significantly. ated with the HbAlc at 8 months. Given that all the participants at 8 months were using the $\mathrm{BC}$, there was no option to differentiate by treatment group.

In the $\mathrm{Co} \rightarrow \mathrm{Cb}$ group, after having used the $\mathrm{BC}$ for 4 months, postprandial hypoglycemia episodes were significantly reduced. This group also had at 8 months less total and postprandial hypoglycemia and $<50 \mathrm{mg} / \mathrm{dL}$ than the $\mathrm{Cb}$ group (Table 1). Again for the whole sample, if we divided it into two groups, participants that just before using BC had a HbAlc $\geq$ or $<8.5 \%$, hypoglycemic events behaved differently. After using the BC for 4 months, the reduction in the total hypoglycemic events reached significance for participants with a $\mathrm{HbA} 1 \mathrm{c}<8.5 \%(7.71 \pm 4.70$ vs. $6.00 \pm 4.21 / 2$ weeks, $P 0.03$ ). For participants with a HbA1c $\geq 8.5 \%$ before using $\mathrm{BC}$ it showed a trend toward reduction, not reaching significance $(6.76 \pm 4.35$ vs. $5.48 \pm 3.63 / 2$ weeks, $P$ 0.10).

Table 3. Linear Regression Model HbA1C at 8 Months

\begin{tabular}{lcccccc}
\hline Model & $\mathrm{B}$ & SE & Beta & $\mathrm{t}$ & $\mathrm{P}$ & Adj $R^{2}$ \\
\hline $\begin{array}{l}\text { HbA1c } \\
\quad \text { 4 months }\end{array}$ & 0.723 & 0.096 & 0.666 & 7.517 & 0.000 & 0.530 \\
$\begin{array}{l}\text { No. of SMBG } \\
\text { tests }\end{array}$ & -0.144 & 0.066 & -0.192 & -2.167 & 0.034 & \\
\hline
\end{tabular}

Group of treatment, sex, age, basal HbA1c, level of education, No. of hypoglycemic episodes at 4 months, and BMI did not enter in the model significantly. SE, standard error. 
Table 4. Linear Regression Model No. of Hypoglycemic Episodes at 8 Months

\begin{tabular}{|c|c|c|c|c|c|c|}
\hline Model & $\mathrm{B}$ & Std err & Beta & $\mathrm{t}$ & $\mathrm{P}$ & $\operatorname{Adj} R^{2}$ \\
\hline No. of hypoglycemic episodes, basal & 0.270 & 0.098 & 0.300 & 2.752 & 0.008 & 0.588 \\
\hline Duration of DM (years) & -0.149 & 0.048 & -0.335 & 3.126 & 0.003 & \\
\hline Treatment group & 2.417 & 0.905 & -0.289 & 2.671 & 0.010 & \\
\hline
\end{tabular}

Alcohol and ISF did not enter in the model significantly.

DM, diabetes mellitus.

In a regression model, with the number of hypoglycemic episodes at 8 months as the dependent variable, the number of basal hypoglycemia, the evolution of DM (in years), and the treatment group formed a significant part of the model explanation (Table 4).

During this phase, three episodes of severe hypoglycemia (in two participants of the Cb group) and two moderate hypoglycemia (one in the $\mathrm{Cb}$ group and one in the $\mathrm{Co} \rightarrow \mathrm{Cb}$ group) were recorded. None of the subjects affected had a similar precedent during the year before the study. During the second phase of the study nine participants with precedents of such hypoglycemic episodes in the previous year (five in the $\mathrm{Cb}$ group and four in the Co group) completed the study period without having suffered from severe or moderate hypoglycemic episodes.

Regarding major hyperglycemia events $(>250 \mathrm{mg} / \mathrm{dL})$, no differences were observed between groups or within them at 8 months (Table 1).

Only for the $\mathrm{Cb}$ group the $\mathrm{SD}$ of glycemia was slightly but significantly increased from the fourth to the eighth months. Estimations of glycemic variability such as MAGE, LBGI, or HBGI did not show any significant changes during the study, either between or within groups (Table 1).

A reduction in the number of postprandial SMBG tests at the end of the study regarding baseline was observed for both groups, although it was only significant in the group that used $\mathrm{BC}$ for 8 months ( $\mathrm{Cb}$ group, baseline $2.07 \pm 0.59$ per day, vs. 8 months $1.59 \pm 0.72, P<0.001)$.

The mean CarbF for breakfast, lunch, and dinner was, respectively, $1.34 \pm 0.64,1.17 \pm 0.46$, and $1.16 \pm 0.43 \mathrm{IU} / \mathrm{KE}$ before using the $\mathrm{BC}$ and $1.50 \pm 0.69,1.10 \pm 0.52$, and $1.18 \pm 0.44$ at the end of the study. These differences were significant for breakfast $(P$ 0.002). The mean ISF before using the $\mathrm{BC}$, for the same meals, was, respectively, $45.88 \pm 8.82$, $45.61 \pm 10.26$, and $46.06 \pm 9.3 \mathrm{mg} / \mathrm{dL}$ and $40.86 \pm 13.99$, $43.72 \pm 12.28$, and $42.83 \pm 13.42$ at the end of the study. These differences were also significant for breakfast $(P$ 0.005).

For all the participants, when comparing the distribution of the CarbF from before using the BC to the end of the study, significantly more subjects switched to an increase of the CarbF ( >1) for breakfast and showed a trend to decrease the CarbF for lunch; the ISF was also significantly reduced $(<50)$ for breakfast and dinner for the same period (Fig. 2).

The treatment satisfaction improved significantly in the group that had been using the BC for all the 8 months compared with the group that had used it only for 4 months; regarding the fear of hypoglycemia, a significant improvement was observed compared with baseline only in the $\mathrm{Cb}$ group (Fig. 3). The use and acceptance of the BC got high scores at the end of the study for all the asked items (Fig. 4).

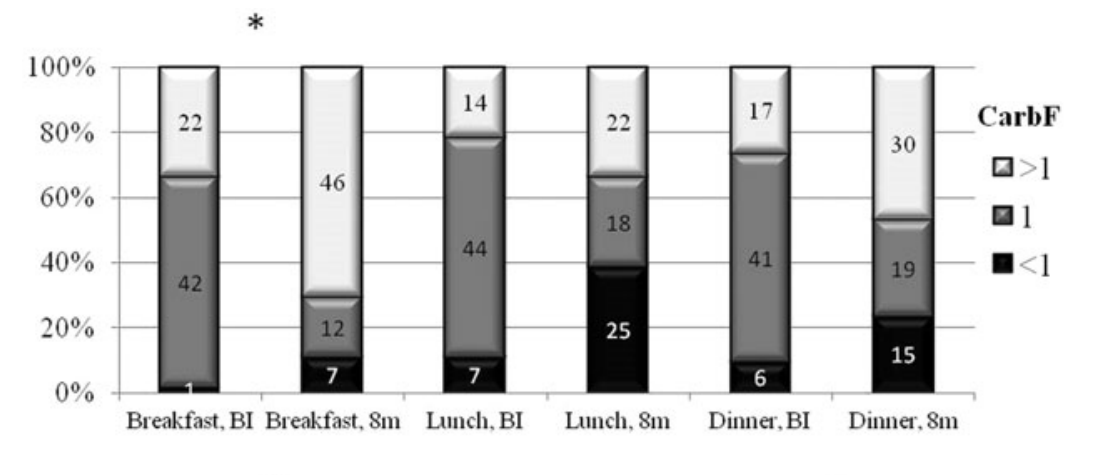

$*$

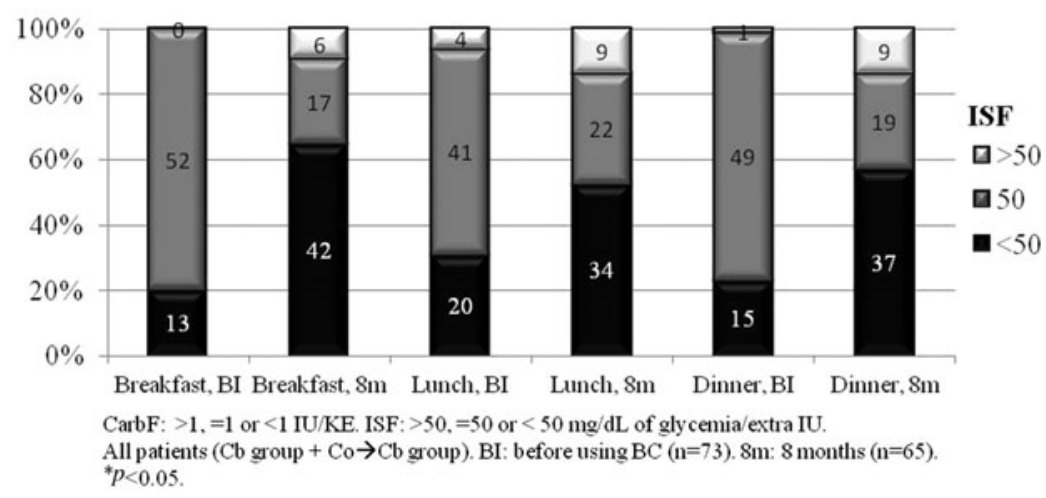

FIG. 2. Changes in CarbF and ISF from before $\mathrm{BC}$ to the end of the study ( 8 months). BC, bolus calculator; ISF, insulin sensitivity factor. 

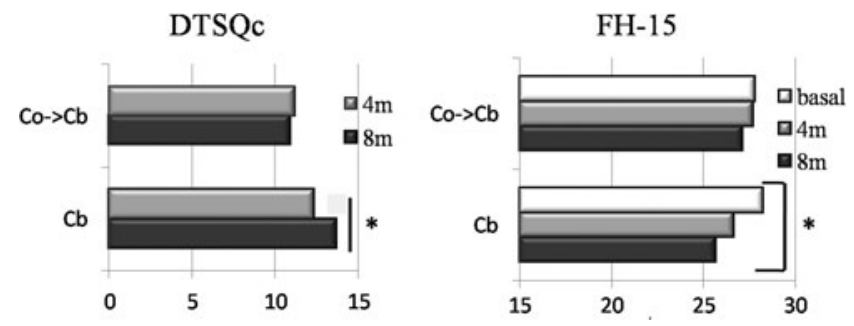

FIG. 3. DTSQc (Diabetes Treatment Satisfaction Questionnaire-change) and FH-15 (Fear of Hypoglycemia). $4 \mathrm{~m}, 4$ months; $8 \mathrm{~m}, 8$ months. ${ }^{*} P<0.05$.

Twenty-one participants $(24 \%)$ were lost (dropout and withdrawal) during the follow-up, most of them $(n=16)$ in the first phase of the study. Twelve belonged to the Co group and 9 to the $\mathrm{Cb}$ group, without overall differences between groups, but most participants from the Co group leaving the study did it in the first phase ( $n=11 / 12)$ (Fig. 1).

No differences were found in age, sex, and duration of DM in the patients who completed the study compared with those who did not. Basal HbA1c was significantly higher in the participants who left the study $(8.71 \% \pm 1.02 \%$ vs. $8.28 \% \pm 0.80 \%, P 0.048)$; they also had higher basal mean total and preprandial glycemia $(180.28 \pm 43.06 \mathrm{mg} / \mathrm{dL}$ vs. $164.27 \pm 23.67 \mathrm{mg} / \mathrm{dL}, P \quad 0.047 ; 177.33 \pm 46.88 \mathrm{mg} / \mathrm{dL}$ vs. $158.54 \pm 25.39 \mathrm{mg} / \mathrm{dL}, P 0.034)$ and performed less daily SMBG tests at baseline $(4.74 \pm 1.38$ vs. $5.37 \pm 1.02, P 0.043)$.

\section{Discussion}

People with DM1 treated with flexible insulin treatment face the challenge of performing complicated calculations before deciding what insulin bolus dose to take. This process includes the application of concepts such as the CarbF, ISF, and residual active insulin. ${ }^{15}$ BCs facilitate these calcula- tions; however they require training, reprogramming, followup, and confidence in the proposed insulin dose. Several studies have shown the impact of this technology on the psychological well-being and metabolic control of people with DM1 treated with MDI. ${ }^{3-5}$

In the first phase of our study we had demonstrated a significant decrease in $\mathrm{HbA} 1 \mathrm{c}$ for the group treated with $\mathrm{BC}$ during 4 months $(-0.66 \%)$ and also for the active control group $(-0.39 \%) .{ }^{10}$ Although HbAlc or its decrease was not significantly different between groups in the bivariate comparisons, in a linear regression model the treatment group was significant to explain the decrease of the $\mathrm{HbAlc}$. Besides that, the group treated with $\mathrm{BC}$ significantly reduced hypoglycemic episodes. ${ }^{10}$

During this second phase of the study the implementation and use of BCs in the control group during 4 months did not produce any additional improvement in the glycemic control compared with the previous period. Starting out from an intensified treatment, it was probably going to be difficult to achieve any additional improvement. In the group that had been wearing the $\mathrm{BC}$ for all the 8 months of the study there was not a further reduction in the $\mathrm{HbAlc}$, rather it was only maintained. Probably the effect of any treatment intensification on glycemic control could be demonstrated in the short term, but at medium term only its maintenance can be expected. ${ }^{11}$ The ABACUS study, which was 26 weeks long, is the one that was closest to ours in terms of time; they started out with a higher basal HbA1c (8.9\% vs. $8.4 \%$ in our study) meaning that its decrease with intervention was potentially more feasible, ${ }^{5}$ due to the known fact that initial HbAlc has an influence on the change. ${ }^{16}$ This is supported by the fact that, in our study, HbA1c improved mainly in the subgroup of participants that had a figure $\geq 8.5 \%$ before using BC.

The incorporation of the $\mathrm{BC}$ in the control group led to a decrease of postprandial hypoglycemic episodes, just as we

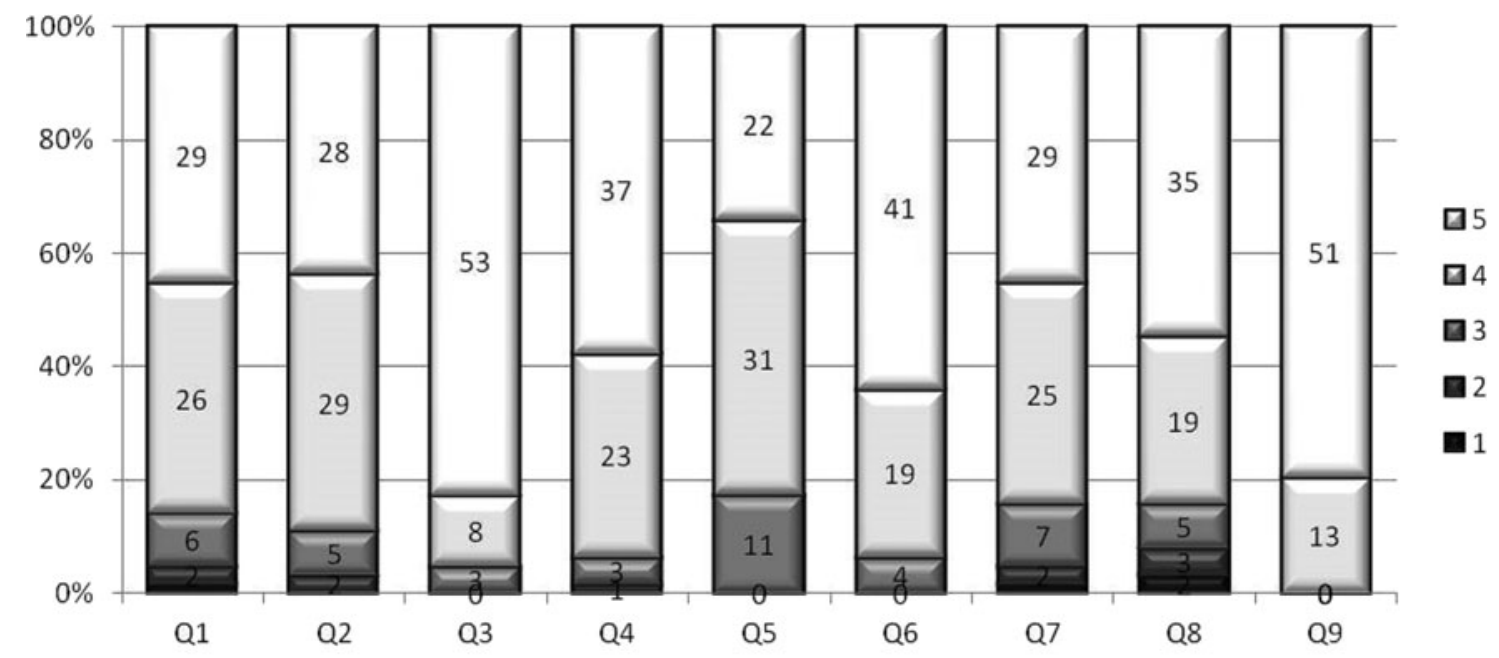

Q1: the use of this device is easy to handle; Q2: I am confident using this device; Q3: I routinely use the device; Q4: the use of this device gives me better control of my diabetes; Q5: the use of this device gives me better postprandial control; Q6: the use of this device is better than performing mental calculations; Q7: it is easy to learn how to use the device; Q8: the benefits of using the device outweigh the time and effort required to learn how to use it; and Q9: I used the device frequently over the past 2 weeks. Expressed as $n$ and proportions.

FIG. 4. Acceptance questionnaire about using the BC (all subjects, 8 months). 
found in the first phase of the study, when the total hypoglycemia was reduced in the $\mathrm{Cb}$ group ${ }^{10}$; that is to say, in both groups the incorporation of the $\mathrm{BC}$ achieved some reduction in hypoglycemia. This improvement was found mainly for the subgroup of participants on a better basal metabolic control $(\mathrm{HbA} 1 \mathrm{c}<8.5 \%)$. In the regression study, the type of treatment proved to be significant in explaining the hypoglycemia. It is possible that the hypoglycemic episodes were reduced due to a better individualization of ISF and because the BC programming takes the active insulin into account; both could have avoided overdoses of corrective insulin. In the Expert study, ${ }^{6}$ performed in our area also in subjects with DM1 on treatment with MDI, a reduction in postprandial hypoglycemia was also found. Another Study in a pediatric population also showed a reduction in hypoglycemia. ${ }^{17}$ The ABACUS study on the contrary showed more total hypoglycemia in the group that used the BC. ${ }^{5}$ In the BolusCal study no differences for hypoglycemia were found between treatment groups. ${ }^{4}$ Historically, the risk of many forms of treatment intensification in DM is the increase in the rate of hypoglycemia episodes, mostly severe ones. ${ }^{18}$ Therefore the $\mathrm{BC}$ can be considered to be a useful tool for treatment intensification without increasing, or even (according to our study) achieving a discreet but significant reduction in hypoglycemic episodes.

With the use of the BC, contrary to what could be expected, the subjects reduced the number of SMBG tests, and less postprandial SMBG tests were recorded than the number requested from the study protocol. The postprandial glycemia allegedly would be modifiable or more affected by the introduction of a BC and could then be biased; probably the patients performed fewer postprandial SMBG tests because they thought they were being well controlled and therefore they did not deem it necessary, limiting the SMBG tests to less representative moments, this could be, when hyperglycemia or hypoglycemia was suspected. Including a larger group of participants, a higher number of visits or the use of a CGM (continuous glucose monitor) could have provided more data, but the latter could not be included in the study due to the lack of funding. In the ABACUS study the number of daily SMBG tests were maintained throughout the study in both groups ${ }^{19}$; in the Expert study, in which also a continuous glucose monitoring system was used, the number of daily SMBG tests increased significantly with the use of BC. ${ }^{6}$

In the subjects from the $\mathrm{Cb}$ group, at the eighth month, the glycemic variability measured by SD increased compared with the fourth month. This could be due to the slight decrease in the number of SMBG tests, which could have been performed more often in situations when hyper- or hypoglycemia was suspected, thereby explaining the greater dispersion in the glycemia obtained. The ABACUS study described a reduction in MAGE in people treated with $\mathrm{BC}^{5}$ while in the Expert study no significant differences were found regarding glycemic variability. ${ }^{6}$

The CarbF throughout the study was increased significantly for breakfast. Regarding ISF, it was reduced throughout the study to nearly $40 \mathrm{mg} / \mathrm{dL}$, figure we suggest to be applied more widely.

In the participants who used the $\mathrm{BC}$ during all the study $(\mathrm{Cb}$ group) a significant reduction in the fear of hypoglycemia was observed at 8 months compared with the basal situation. Only in one study, performed also in people with DM1 on treatment with MDI, a similar improvement was found. ${ }^{3}$ In the BolusCal study no differences in the fear of hypoglycemia were proved, ${ }^{4}$ but the questionnaire used was the Hypoglycemic Fear Scale (HFS), different to the one used in our study (FH-15). The analysis of the fear of hypoglycemia in the ABACUS study has not been published yet. ${ }^{5}$ In the Expert study fear of hypoglycemia was not evaluated. ${ }^{6}$

At the end of our study (8 months) an improvement in treatment satisfaction was observed only in the group that had been using the $\mathrm{BC}$ from the start ( $\mathrm{Cb}$ group). Similar results were also observed in other studies with BC and CSII ${ }^{2,20,21,22}$ or MDI. ${ }^{4,5}$

The losses in the Co group occurred mostly early in the study, and in the $\mathrm{Cb}$ group later, during the follow-up. The initial dropouts could be due to the disappointment for not having been included in the $\mathrm{Cb}$ group from the outset of the study, which concurs with the interpretation of the investigators in the ABACUS study. ${ }^{9}$ There were no dropouts or withdrawals due to the difficulty of using the device, in fact, the participants considered the BC easy to learn and use, as can be seen in the scores of the questionnaires on the use of the BC.

Among the limitations of this study we highlight the high percentage of dropouts. The group that did not complete the study, irrespective of the moment of dropping out, presented a worse metabolic control at baseline, with a higher HbAlc. These types of subjects are those who could theoretically further improve HbA1c with any type of intervention. ${ }^{16}$ The highest percentage of dropouts that initially affected the Co group could have biased this group toward the more reliable participants. In contrast some participants performed few postprandial SMBG and others reduced them during the study, thereby making the reprogramming of the CarbF and ISF more difficult to perform, which could have also negatively affected the potential efficiency of the BC on metabolic control and hypoglycemia. As strength, we could highlight the prospective randomized and controlled design of our study and that its duration ( 8 months, greater than other studies) has enabled us to prove psychological benefits than were not shown in the short term and to verify that the improvement obtained in the HbA1c can be sustained over time.

In conclusion, compared with the first part of the study (in which both the group treated with $\mathrm{BC}$ and the control group improved their glycemic control), the extension of the follow-up to 8 months could not obtain a greater benefit concerning glycemic control (HbA1c), but rather could only maintain it. As had been observed in the group who used the $\mathrm{BC}$ in the first 4 months of the study, the control group that was incorporated in the use of the device also achieved a significant reduction in hypoglycemic episodes. Furthermore, in the subjects in which the use of the BC was extended to 8 months, a greater treatment satisfaction was observed and likewise a reduction in the fear of hypoglycemia. The subjects considered the $\mathrm{BC}$ to be a well valued tool which they could trust and they used it to make the majority of their decisions concerning insulin bolus.

\section{Acknowledgments}

This project was partially funded by the Sociedad Andaluza de Endocrinología y Nutrición (SAEN). No other funding was received. The authors thank Roche Diagnostics (Barcelona, Spain) for providing the Accu-Chek ${ }^{\circledR}$ Aviva Expert and Accu-Chek Aviva Nano devices for the study. The authors also thank the nurses M. Fontalba and $\mathbf{M}$. 
Guerrero (Investigation Unit, Endocrinology and Nutrition Department, Hospital Regional Universitario de Málaga) for their contribution to the trial and C. Corazza for her kind review of the text.

\section{Author Disclosure Statement}

M.R.V.M. and S.G.R. have received funding from Roche Laboratories to attend courses and conferences. The Endocrinology and Nutrition Department of the Hospital Regional Universitario, and specifically S.G.R. as the main investigator, participated in the ProAct study (Accu-Chek ${ }^{\circledR}$ Aviva Combo on insulin pumps treatment), which was unrelated to the financial support received for the present study. In 2012, Roche Diabetes Care (Barcelona, Spain) made a contribution for generic investigations into diabetes to the Endocrinology and Nutrition Department, through the Maimonides Investigation Foundation, that was used to hire a nurse for 1 year who worked on several diabetes related projects.

\section{References}

1. Ahola AJ, Makimattila S, Saraheimo M, et al.: Many patients with type 1 diabetes estimate their prandial insulin need inappropriately. J Diabetes 2010;2:194-202.

2. Schwartz FL, Shubrook JH, Marling CR: Use of case-based reasoning to enhance intensive management of patients on insulin pump therapy. J Diabetes Sci Technol 2008;2:603611.

3. Barnard K, Parkin C, Young A, et al.: Use of an automated bolus calculator reduces fear of hypoglycemia and improves confidence in dosage accuracy in patients with type 1 diabetes mellitus treated with multiple daily insulin injections. J Diabetes Sci Technol 2012;6:144-149.

4. Schmidt S, Meldgaard M, Serifovski N, et al.: Use of an automated bolus calculator in MDI-treated type 1 diabetes: the BolusCal Study, a randomized controlled pilot study. Diabetes Care 2012;35:984-990.

5. Ziegler R, Cavan DA, Cranston I, et al.: Use of an insulin bolus advisor improves glycemic control in multiple daily insulin injection (MDI) therapy in patients with suboptimal glycemic control. First results from the ABACUS trial. Diabetes Care 2013;36:3613-3619.

6. Gonzalez C, Picón MJ, Tomé M, et al.: Expert Study: utility of an automated bolus advisor ssystem in patients with type 1 diabetes treated with multiple daily injections of insulin-a crossover study. Diabetes Technol Ther 2016; 18:282-287.

7. Cavan DA, Ziegler R, Cranston I, et al.: Use of an insulin bolus advisor improves glycemic control in poorly controlled diabetes: first results from the ABACUS trial. Diabetes Technol Ther 2013;15 Suppl. 1:A101.

8. Cavan DA, Ziegler R, Cranston I, et al.: Study protocol. Automated bolus advisor control and usability study (ABACUS): does use of an insulin bolus advisor improve glycemic control in patients failing multiple daily injection (MDI) therapy? BMC Fam Pract 2012;13:102.

9. Parkin C, Barnard K, Deborah A, et al.: Safe and efficacious use of automated bolus advisors in individuals treated with multiple daily insulin injection (MDI) therapy: lessons learned from the automated bolus advisor control and usability study. J Diabetes Sci Technol 2015;9:1138-1142.

10. Vallejo-Mora MR, Carreira Soler M, Linares Parrado F, et al.: The calculating boluses on multiple daily Injections
(CBMDI) study: a randomized controlled trial on the effect on metabolic control of adding a bolus calculator to multiple daily injections in people with type 1 diabetes. $J$ Diabetes 2016;9:24-33.

11. Ziegler R, Tubili C, Chico A, et al.: ProAct study: new features of insulin pumps improve diabetes management and glycemic control in patients after transition of continuous subcutaneous insulin infusion systems. Diabetes Technol Ther 2013;15:738-743.

12. Gomis R, Herrera-Pombo JL, Calderón A, et al.: Validación del cuestionario "Diabetes Treatment Satisfaction Questionnaire" (DTSQ) en la población española. Pharmacoeconomics 2006;3:7-18.

13. Bradley C: Diabetes treatment satisfaction questionnaire. In: Bradley C, ed. Handbook of Psychology and Diabetes. Chur, Switzerland: Harwood Academic Publishers, 1994.

14. Anarte M, Caballero FF, Ruiz de Adana MS, et al.: Development of a new Fear of Hypoglycemia Scale: FH 15. Psychol Assess 2011;23:398-405.

15. Zisser H, Robinson L, Bevier W, et al.: Bolus calculator: a review of four "smart" insulin pumps. Diabetes Technol Ther 2008; 10:441-444.

16. Schmidt S, Norgaard K: Bolus calculators. J Diabetes Sci Technol 2014;8:1035-1041.

17. Ramotowska A, Szypowska A: Bolus calculator and wirelessly communicated blood glucose measurement effectively reduce hypoglycaemia in type 1 diabetic children-randomized controlled trial. Diabetes Metab Res Rev 2014;30:146-153.

18. Misso ML, Egberts KJ, Page M, et al.: Continuous subcutaneous insulin infusion (CSII) versus multiple insulin injections for type 1 diabetes mellitus. Cochrane Database Syst Rev 2010;1:A107-A108.

19. Cavan D, Ziegler R, Cranston I, et al.: Use of an insulin bolus advisor facilitates earlier and more frequent changes in insulin therapy parameters in suboptimally controlled patients with diabetes treated with multiple daily insulin injection therapy: results of the ABACUS trial. Diabetes Technol Ther 2014;16:310-316.

20. Gross TM, Kayne D, King A, et al.: A bolus calculator is an effective means of controlling postprandial glycemia in patients on insulin pumps therapy. Diabetes Technol Ther 2003;5:365-369.

21. Klupa T, ek-Klupa T, Malecki M, et al.: Clinical usefulness of a bolus calculator in maintaining normoglycaemia in active professional patients with type 1 diabetes treated with continous subcutaneous insulin infusion. J Int Med Res 2008;36:1112-1116.

22. Shashaj B, Busetto E, Sulli N: Benefits of a bolus calculator in pre- and postprandial glycaemic control and meal flexibility of paediatric patients using continuous subcutaneous insulin infusion (CSII). Diabetic Med 2008;25:1036-1042.

Address correspondence to: María del Rosario Vallejo Mora, PhD, MD Endocrinology and Nutrition Department Hospital Regional Universitario de Málaga Pabellón C (Hospital Civil) Plaza del Hospital Civil Málaga 29009 Spain

E-mail: rosariovallejomora@hotmail.com 